mit den Bildwerken anderer Länder dürfen Schlüsse in hastigem Generalisieren nicht gezogen werden. Wohl aber ist auch weiter den Parallelen nachzugehen. Vor allem aber gilt auch hier, was für die gesamte Wissenschaft vom alten Jemen gilt: es muss endlich einmal an Ort und Stelle fachmännisch gearbeitet werden, um zu retten, was noch zu retten ist.

\section{Die Statue des Priesters Sethon zn Memphis.}

Von A. Wiedemann.

Herodot II 141 erzählt, der Priester des Hephaestos Sethon sei in einem Kriege gegen die Assyrer durch Mäuse gerettet worden und gibt dabei eine Volkssage über die auch im Alten Testamente erwähnte, vermutlich durch eine Lagerpest erfolgte Vernichtung des Heeres des Sanherib bei seinem dritten Feldzuge gegen Vorderasien wieder. Seinen Bericht knüpft er an den "noch jetzt" in dem Tempel des Hephästos stehenden steinernen König, der auf der Hand eine Mans hatte. Ich selbst habe früher') bei dieser Statue an ein Bildnis des Gottes Horus mit der ihm heiligen Maus gedacht. Spiegelberg'), der die literarische Bedeutung der Sage behandelte, schlug vor, in ihm einen Hohenpriester von Memphis $\mathrm{zu}$ 'sehen, der durch die Maus auch als Hoherpriester des Gottes von Letopolis bezeichnet worden wäre. Oestrup ${ }^{3}$ ) sah in dem Bildwerk den Pestgott; die Inschrift, die nach Herodot die Statue trug: "Wer mich anblickt, der soll $\varepsilon \dot{v} \sigma \varepsilon \beta \dot{\eta} \varsigma^{\prime \prime}$, von der Strafe des Gottes unberührt "sein": zeige, dass der Aegypter hier ähnlich dachte, wie der Israelit, wenn er die eherne Schlange anblickte. Demgegenüber betonte Spiegelberg ${ }^{4}$ ), dass man eine derartig von Herodot gegebene Inschriftsdeutung nicht $\mathrm{zu}$ ernst nehmen dürfe.

So richtig dieser Einwand auch im Prinzip ist, ganz verwerfen möchte ich die Herodoteische Angabe doch nicht und in ihr wenigstens einen Hinweis darauf sehen, dass dem Gewährsmann, der Herodot die Statue zeigte, das Bildnis eine apotropäische, Uebel abwehrende Bedeutung zu haben schien. Man

1) Herodots Zweites Buch, S. 504 .

2) Aeg. Zeitschr. XLIII, S. 92. s. 868 .

Orientalische Studien, Nöldeke gewidmet II hat, wie auch Oestrup, meist auf Grund semitischer und klassischer Anschauungen die Maus mit der Pest und dem diese abwehrende Apollo Smintheus in Verbindung gebracht. Allein eine derartige Auffassung ist bisher für das alte Aegypten nicht nachgewiesen und erscheint es daher zunächst geratener, zu versuchen, ob sich nicht mit Hilfe einer ägyptischen Ansicht über die Maus und ihre religiöse Bedeutung etwas zur Klärung der Frage beibringen lässt.

Eine Reihe antiker Autoren, unter denen freilich Herodot selbst fehlt, Diodor, Aelian, Plinius, Mela, Plutarch, Macrobius ${ }^{1}$ ) berichten, in Aegypten entständen Mäuse aus Erdschollen, man fände gelegentlich Tiere, deren Vorderkörper sich bereits ausgebildet habe, während der Rest noch Erde sei ${ }^{2}$ ). Dieser Glaube hat die Jahrtausende überdauert; noch nach der heutigen ägyptischen Volksmeinung entstehen die Ratten aus dem Nilschlamm ${ }^{3}$ ). Die ganze Legende wird wohl zunächst aus der eigenartigen Gestalt der ägyptischen Wüstenspringmaus entstanden sein. Mit seinen langen Hinterbeinen, dem langen Schwanz und dem im Gegensatz zu Kopf und Vorderbeinen stark entwickelten Hinterleib kann dieses Tier leicht den Eindruck eines nicht fertig entwickelten Wesens hervorrufen. Ebenso wie die Mäuse entstehen nach Plinius, Hist. nat. IX. 74, die Frösche aus dem Schlamm, und mit dieser Vorstellung hängen die zahlreichen Stellen ${ }^{4}$ ) zusammen, an denen der Frosch als ein Symbol der Auferstehnng aufgefasst wird.

Auf einer magischen Stele des Museums zu Kairo ${ }^{5}$ ) steht neben zahlreichen anderen Uebel abwehrenden Dämonen ein kleiner zwergartiger Mann in der Gestalt der Chnumu. In jeder Hand hält er eine Art Band, während auf seinem Kopfe ein im Profil gezeichnetes langschwänziges mausartiges Tier sitzt. Denselben Chnumu mit der Maus auf dem Kopfe stellt vielleicht auch ein Dämon auf der Metternich-Stele ${ }^{6}$ ) dar, welcher in jeder

1) Wiedemann, Herodots Zweites Buch, S. 290. Vgl. ferner Ovid. Metam. I. $422 \mathrm{ff}$.

3) Ueber die rattenartigen Tiere in den ägyptischen Texten rgl. Lefébure, Sphinx VI, S. $189 \mathrm{ff}$., VII, S. $25 \mathrm{ff}$. Gutes naturalistisches Bild der Springmaus aus Meïr bei Legrain, Ann. du Bervice I, S. 71 , shnlich die Abbildung Brebms Tierleben $\mathrm{II}^{2}, \mathrm{~S}$. 332 . 3) Maspero, Hist. anc. des peuples de l'Orient classique I, S. 156, Anm. 6.

4) Jacoby und Spiegelberg, Sphinx VII, S. 215 ff., VIII, S. $78 \mathrm{f}$.

5) Nr. 9402 Revers bei Daressy, Textes et Dessins magiques (Cat. gén. du Musée du Caire), S. $3 \mathrm{ff}$, pl. III. 6) Ed. Golenischeff, Taf. V, No. XXIII. 
Hand eine Schlange hält und ein unklares Zeichen auf dem Haupte trägt.

Die Chnumu gehören zu den Gottheiten, welche einerseits das Böse verscheuchen, andererseits das Wiederaufleben nach dem Tode, bez. die Wiederherstellung des irdischen Leibes erleichtern. Sie laufen mit dem Gotte Bes parallel, dem die gleiche doppelte Bedeutung zugeschrieben wird; und man legt daher die Tonstatuetten beider Gestalten gern den Toten in das Grab. Auch architektonisch werden beide gleichartig verwendet. In einem Tempel zu Gebel Barkal aus der Zeit des Taharka, also etwa aus derselben Periode, in welcher Sethon gelebt haben soll, lehnen die Chnumu in gleicher Weise an den Pfeilern ${ }^{1}$ ), wie an gleicher Stelle und in mehreren Tempeln des ausgehenden Neuen Reiches im eigentlichen Aegypten der Gott Bes²). Hier sollen sie in erster Reihe, wie die Bilder der geflügelten Sonnenscheibe, der Hathormaske usf. Uebel abwehrend wirken. Nebenbei aber werden sie gleichzeitig, wie die Osiris-Bilder an den Pfeilern der Totentempel, nach dem Tode des Königs, der den Tempel weihte und dem hier ein Teil des Kultes galt, an seiner Wiederbelebung Anteil zu nehmen haben.

Kommt den Chnumu eine solche Bedeutung zu, so wird dieselbe auch der Maus, die mit einem von ihnen auf der Kairener Stele verbunden erscheint, zuzuschreiben sein. Die Mäuse entstanden, wie eben ausgeführt, ebenso wie die Frösche, selbständig aus dem vom Nile befruchteten Schlamm. Sie mussten daher so gut wie diese als Symbole und als Verbürger des neuen Lebens nach dem Tode, der Auferstehung, gelten. War doch die Generatio aequivoca, die selbst Aristoteles ${ }^{3}$ ) noch beim Aal, bez. bei Würmern für möglich hielt, das beste Analogon und damit der beste magische Parallelvorgang für die Neugeburt der Menschen aus seinem toten Leichnam.

Man könnte zunächst aus Herodots Schilderung schliessen, die Statue habe ähnlich ausgesehen wie ein bekanntes Bildnis des Bes, bei dem der Gott den sitzenden Hundskopfaffen auf der ausgestreckten rechten Hand hält, während er mit der linken das auf seiner Schulter sitzende Kind stützt4).

1) Leps. Denkm. I, 127; V, 6c.; Maspero, Hist. anc. de l'Orient classique III, S. 365 nach Caillaud, Voy. à Méroé. I, pl. 74 .

2) Vgl. für diesen Gott Krall in Benndorf, Heroon von Gjoelbaschi-Trysa, S. $72 \mathrm{ff}$.

9) Hist. anim. VI, 15.

4) Statuette bei Bonomi und Arundale pl. 23
Allein, in diesem und in ähnlichen Fällen handelt es sich um kleine Statuetten, an die kaum eine Volkssage angeknüpft haben wird. Als Ausgangspunkt für eine solche wird man eher an eine imponierend grosse Gestalt $\mathbf{z u}$ denken haben. Bei dieser aber erscheint, wenn sie, wie Herodot angibt, aus Stein gearbeitet war, das Vorstrecken der Hand wenig wahrscheinlich, da eine solche Haltung die Standsicherheit, auf die die Aegypter stets grosses Gewicht legten, stark gefährdet haben würde. So wird das Bildwerk eher die eine Hand auf die Brust gelegt und auf dieser die Maus getragen haben, ähnlich wie andere Statuen in dieser Stellung das Ut'a-Auge halten 1).

Da von der Anlage des Ptah-Tempels zu Memphis nur sehr wenig bekannt ist, wird man schwanken können, ob es sich bei dem Bildnis um eine isolierte Statue handelt oder um eine aus einer Reihe von Götterpfeilern. Denn das wird man vermuten müssen, dass, wenn sich im PtahTempel ein offener Hof mit Götterpfeilern befand, dass dann an diesen Bilder der Genossen des Ptah, der Chnumu, als Uebelabwehrer und nebenbei als Unsterblichkeitsverbürger angebracht waren. Eines von diesen hätte dann dem in dem Tempel aufgestellten frommen Sethon Herodots entsprochen.

\section{Bespreehungen.}

Vorderasiatische Bibliothek. I. Band, Abteilung 1. Die sumerischen und akkadischen Königsinschriften, bearbeitet von F. Thureau-Dangin, Leipzig J. C. Hinricbssche Buchhandlung 1907. $\mathrm{XX}$, $275 \mathrm{~S}$. Besprochen von Bruno Meissner.

Die keilinschriftliche Bibliothek, die seiner Zeit grossen Nutzen gestiftet hat, aber langsam vorwärts schreitet und nur eklektisch verfährt, soll jetzt durch die VAB ersetzt werden. In langer Reihe sollen alle babylonischen und assyrischen Königsinschriften und die wichtigsten Privaturkunden umschrieben und übersetzt werden. Ein auf S. VI veröffentlichter Plan zeigt den Umfang der $\mathrm{zu}$ behandelnden Gebiete und gibt

No. 83 und Pleyte, Chapitres supplémentaires. Traduction chap. 162, 162*, 163. Taf. zu S. 119. Aebnlich halt bei Lanzone, Diz. di mitol. Taf. 198 No. 3 der Nil als Zeichen seines neuen Lebens (vgl. Spiegelberg, Sphinx VII, S. 219) einen Frosch auf der Hand.

1) Vgl. z. B. Daressy, Statues de divinités (Cat. gén. du Musée du Caire) No. 38038, pl. 4. 